\title{
Epigenetic reprogramming of embryos derived from sperm frozen at $-20^{\circ} \mathrm{C}$
}

\author{
CHAO ShiBin ${ }^{1,2}$, LI JianChun ${ }^{1}$, JIN XuanJin ${ }^{1}$, TANG HaiXun ${ }^{1}$, \\ WANG GongXian ${ }^{1} \&$ GAO GuoLan ${ }^{2 *}$ \\ ${ }^{1}$ Center of Reproductive Medicine, the First Affiliated Hospital of Nanchang University, Nanchang 330006, China; \\ ${ }^{2}$ Department of Obstetrics and Gynecology, Aviation General Hospital of China, Beijing 100101, China
}

Received February 21, 2012; accepted March 26, 2012

\begin{abstract}
Cryopreservation of spermatozoa is a strategy that has been used to conserve the sperm of animal species and animal strains that are valuable for biomedical research. A simple method for preserving spermatozoa after application of intracytoplasmic sperm injection (ICSI) is much needed. It has been shown previously that spermatozoa frozen at $-20^{\circ} \mathrm{C}$ can activate oocytes and support full-term embryo development. However, epigenetic reprogramming could be affected by the environment and by the in vitro manipulation of gametes. Here, we investigated embryo epigenetic reprogramming including DNA methylation and histone modification, in embryos derived from sperm preserved at $-20^{\circ} \mathrm{C}$ without cryoprotectants. The results showed that although both fertilization and embryo developmental competence were decreased, the dynamic epigenetic reprogramming of embryos derived from frozen sperm was similar to the reprogramming of embryos derived from fresh sperm. The results reported in this study indicate that sperm frozen without cryoprotectant is epigenetically safe for ICSI.
\end{abstract}

spermatozoa freezing, ICSI, epigenetic, histone modification, DNA methylation

Citation: Chao S B, Li J C, Jin X J, et al. Epigenetic reprogramming of embryos derived from sperm frozen at $-20^{\circ} \mathrm{C}$. Sci China Life Sci, 2012, 55: 349-357, doi: 10.1007/s11427-012-4309-8

Many basic studies of mammalian genetics and early development have been done using laboratory mice including genetically engineered mice (transgenesis, gene targeting, and mutagenesis), the number of which is increasing exponentially. The preservation of mouse spermatozoa now plays an important role in basic research and fertility treatment. Male germ cells were the first cell type to be cryopreserved successfully and now spermatozoa preservation in liquid nitrogen $\left(\mathrm{LN}_{2}\right)$ is commonly used in many mammalian species [1-3] including humans [4]. However, mouse spermatozoa cryopreservation methods are unsatisfactory because of the sperms' extreme sensitivity to cold and osmotic effects compared with human spermatozoa [5].

The first successful cryopreservation of mouse sperma-

*Corresponding author (email: gaoguolan361@163.com) tozoa was reported in 1990 [6]. Before the application of the intracytoplasmic sperm injection (ICSI) technique which is another method used to restore fertility, the motility and fertility of freezing/thawing spermatozoa are the most important factors to consider. ICSI is a very effective technique with applications in both assisted reproductive technology (ART) and in the study of fertilization [7,8]. The single sperm used in ICSI need not necessarily be motile or even viable $[9,10]$ and this technique has been used to produce healthy offspring using frozen spermatozoa without cryoprotectants [11]. Accordingly, new methods have recently been developed to preserve spermatozoa. Wakayama and Yanagimachi [12] reported that spermatozoa freezedried and stored at $4{ }^{\circ} \mathrm{C}$ for 3 months or at room temperature for 1 month did not lose their reproductive potential. The freeze-dried method is now used for spermatozoa preserva- 
tion in several species [13-16]; however, the high cost of the freeze-dried equipment limits its extensive use. Another approach that has been used to preserve spermatozoa without freezing is the evaporative drying method $[17,18]$. However, this method destroys the integrity of DNA and does not preserve spermatozoa successfully for more than 3 months at $4^{\circ} \mathrm{C}$.

Until now, the simplest method for cryopreserving mouse spermatozoa was to freeze the spermatozoa in simple medium without cryoprotectants. In one study, living pups were obtained using spermatozoa stored in PBS buffer for 6 months at $4^{\circ} \mathrm{C}$ [19]. Testicular spermatozoa retrieved from the whole bodies of male mice that had been stored frozen $\left(-20^{\circ} \mathrm{C}\right)$ for 15 years also produced normal offspring via ICSI [20]. Simple but effective sperm preservation methods like this one are much needed in some areas, for example in developing countries, where $\mathrm{LN}_{2}$ is not easily accessed.

Previous studies have indicated that because gametes and preimplantation embryos are sensitive to their environment, in vitro manipulation could affect their developmental potential and epigenetics [21]. Epigenetic reprogramming has been reported to play a vital role in embryonic development [22] and several studies have shown that defective epigenetic reprogramming is associated with abnormal fetal growth and diseases such as cancer and diabetes [21,23].

In this study, karyotype and epigenetic reprogramming, including DNA methylation and modification of histones in embryos derived from spermatozoa frozen at $-20^{\circ} \mathrm{C}$, were analyzed from the pronuclear to blastocyst stages to verify the safety of the spermatozoa in epigenetics. To our knowledge, this study is the first time the epigenetic reprogramming of sperm frozen without cryoprotectants has been investigated.

\section{Materials and methods}

Unless otherwise stated, the chemicals used in this research were purchased from Sigma (St. Louis, MO, USA). All animals were maintained in accordance with the Animal Experiment Standard of the First Affiliated Hospital of Nanchang University, China.

\subsection{Preparation of mouse oocytes}

Female ICR mice (8-12 weeks old) were each injected with 7.5 units of pregnant mare serum gonadotropin (PMSG; Ningbo Animal Hormone Factory, China) followed by injection of 7.5 units of human chorionic gonadotropin (hCG; Ningbo Animal Hormone Factory, China) 48 h later. Mature oocytes were collected from the oviducts $14 \mathrm{~h}$ after the hCG injection and were freed from cumulus cells by a 3-min treatment with $0.3 \%$ hyaluronidase in HEPES-buffered human tubal fluid medium (mHTF; SAGE ART-1023, CA, USA). The oocytes were transferred to human tubal fluid
(HTF) medium (HTF; SAGE ART-1020, CA, USA) containing $10 \%$ human serum albumin (HSA; SAGE ART-3001, $\mathrm{CA}$, USA) and were incubated at $37^{\circ} \mathrm{C}$ under $5 \% \mathrm{CO}_{2}$ for up to 90 min before ICSI micromanipulation.

\subsection{Spermatozoa preparation, freezing and thawing}

Spermatozoa were collected from C57BL/B6 male mouse excised cauda epididymis after squeezing them in an $800-\mu \mathrm{L}$ drop of mHTF containing $10 \%$ HSA. A $100 \mu \mathrm{L}$ aliquot of the sperm suspension was transferred to a $0.5 \mathrm{~mL}$ polypropylene microcentrifuge tube. Each vial was tightly capped and placed directly into the $-20^{\circ} \mathrm{C}$ area of an ordinary refrigerator (Haier BCD-602WM; Qindao, China). To thaw the spermatozoa, the vials were removed from the freezer and placed in water at $24-26^{\circ} \mathrm{C}$ for about $10 \mathrm{~min}$.

\subsection{Mouse ICSI}

ICSI was performed using a micropipette attached to a Piezo-electric actuator (model PMAS-CT150; Prime Tech Ltd., Japan), as previously described [9,24,25]. A single spermatozoon was sucked tail first into an injection pipette, and the head was separated from the tail by applying a few Piezo pulses to the head-tail junction. The isolated sperm head was sucked into an injection pipette (6-9 $\mu \mathrm{m}$ inner diameters at the tip), the mature unfertilized mouse oocyte was stabilized using a holding pipette, and its zona pellucida was penetrated by applying several piezo pulses. When the needle had advanced deeply enough into the ooplasm, the oolemma was punctured with a single piezo pulse and the spermatozoa were slowly released into the ooplasm before the pipette was gently removed. After injection, oocytes were washed three times in HTF and incubated under 5\% $\mathrm{CO}_{2}$ at $37^{\circ} \mathrm{C}$.

\subsection{Karyotype analysis}

Chromosomal analyses of the one-cell zygotes were performed according to the method described previously with some modifications $[26,27]$. In brief, oocytes with two pronuclei at $6 \mathrm{~h}$ after injection were transferred into HTF containing $0.1 \mu \mathrm{g} \mathrm{mL}^{-1}$ colcemid to arrest the cells at the first-cleavage metaphase. Nineteen to $21 \mathrm{~h}$ after ICSI, zygotes were treated with a hypotonic solution of $0.9 \%$ sodium citrate for $10 \mathrm{~min}$ at room temperature and exposed to a freshly prepared fixative mixture of methanol and acetic acid $(3: 1)$. Chromosomes were air-dried and stained with filtered Giemsa in phosphate buffer ( $\mathrm{pH}$ 6.8). Samples were examined with a confocal laser scanning microscope (Zeiss LSM 510 META, Germany). Two groups of metaphase chromosomes were observed, one from oocytes and one from sperm. It has been reported that less than $1 \%$ of normal mouse oocytes have abnormal chromosomes [28,29]. Therefore, in this study, all aberrant chromosomes were 
assumed to be of sperm origin.

\subsection{Immunofluorescent detection}

Embryos were collected at each stage at $24 \mathrm{~h}$ (2-cell), $40 \mathrm{~h}$ (4-cell), $45 \mathrm{~h}$ (8-cell), $64 \mathrm{~h}$ (morula), and $86 \mathrm{~h}$ (blastocyst) after ICSI. Embryos were washed in PBS buffer, fixed in PBS with 4\% paraformaldehyde for $20 \mathrm{~min}$, and permeated with $0.2 \%$ Triton X-100 in PBS for 15 min at room temperature. Subsequently, embryos were blocked for $1 \mathrm{~h}$ in a blocking solution containing 2\% BSA and $0.05 \%$ PBSTween20. Samples were incubated with anti-H3K4-trimethylation (diluted 1:500; Millipore, MA, USA) and antiacetyl histone H4k12 antibodies (1:300, Millipore, MA, USA) for $1 \mathrm{~h}$ and washed with $0.05 \%$ PBS-Tween 20 for $30 \mathrm{~min}$. This was followed by incubation at room temperature for 1 $\mathrm{h}$ with a fluorescein isothiocyanate (FITC) conjugated anti-mouse secondary antibody (ZhongShan Goldenbridge Biotechnology, Beijing, China). After several washes in $0.05 \%$ PBS-Tween 20, samples were chromatin-labeled for $10 \mathrm{~min}$ with $10 \mu \mathrm{g} \mathrm{mL} \mathrm{m}^{-1}$ of propidium iodine (PI) and washed again with $0.05 \%$ PBS-Tween 20. Samples were mounted in $90 \%$ glycerol containing $0.1 \mathrm{~mol} \mathrm{~L}^{-1}$ Tris- $\mathrm{HCl}$ (pH 8.0) and 2.3\% 1,4-diazobicyclo-2,2,2-octane (DABCO) and examined with a confocal laser scanning microscope. Each developmental stage was repeated three times and at least 20 embryos were evaluated each time.

For the detection of DNA methylation, embryos were treated with $4 \mathrm{~mol} \mathrm{~L}^{-1} \mathrm{HCl}$ solution at room temperature for $50 \mathrm{~min}$ and then neutralized for $10 \mathrm{~min}$ with $100 \mathrm{mmol} \mathrm{L}^{-1}$ Tris-HCl buffer ( $\mathrm{pH}$ 8.5) before blocking. After several washes with $0.05 \%$ Tween 20 , the embryos were incubated with 5-methylcytidine antibody (diluted 1:100; Abcam, Cambridge, MA, USA). The rest of preparation was performed using the procedures that were used for the H3K4-trimethylation staining.

\subsection{Immunofluorescent quantitative analysis and data analysis}

The fluorescence images were analyzed using the ImageJ software (National Institute of Health, http://rsbweb.nih. gov/ij/). The relative intensity was calculated as described previously [30]. The nuclear intensities of integrated fluo- rescence were measured by manually outlining all nuclei, 15 nuclei per morula and 30 nuclei per blastocyst. We used the merged images corrected for background by subtracting the mean intensity of the cytoplasmic area from the whole image. The nuclear intensities were measured by manually outlining all of the nuclei. The total fluorescence intensity emitted by each individual nucleus was measured using the ImageJ imaging software and the average intensity for each blastomere was calculated using the ratio of the antibody signal to the PI DNA signal.

Differences between two groups were analyzed by ANOVA using SPSS software (SPSS Inc., Chicago, IL, USA) followed by the Student-Newman-Keuls test. The results were considered statistically significant when $P<0.05$.

\section{Results}

\subsection{Fertilization and embryonic development of sperm stored at $-20^{\circ} \mathrm{C}$ without cryoprotectants}

To evaluate the fertility ability of spermatozoa preserved at $-20^{\circ} \mathrm{C}$, the sperm were microinjected into ICR mouse oocytes. Oocytes that presented two polar bodies and two pronuclei $6 \mathrm{~h}$ after ICSI were considered to be fertilized. The fertilized oocytes were incubated at $37^{\circ} \mathrm{C}$ under $5 \%$ $\mathrm{CO}_{2}$. The number of embryos that reached the two-cell and blastocyst stages 24 and $86 \mathrm{~h}$ after ICSI, respectively, were counted. For sperm that had been stored at $-20^{\circ} \mathrm{C}$ for 1 month, we found that $85.2 \%(121 / 142)$ of the oocytes were fertilized, and of these $86.7 \%(105 / 121)$ reached the two-cell embryo stage and $46.3 \%(56 / 121)$ became blastocysts. Compared to the rates in the fresh (control) group, the fertilization rate and the blastocyst rate of the stored sperm were significantly reduced. For sperm stored at $-20^{\circ} \mathrm{C}$ for 6 months, the fertilization and blastocyst rates were even lower (compared to the fresh group, $P<0.01$ ) (Table 1).

\subsection{Chromosome analysis of one-cell zygotes generated by fresh and frozen sperm}

Orly et al. [31] found that there was chromosome damage in spermatozoa frozen in $\mathrm{LN}_{2}$ without cryoprotectants. In the

Table 1 Fertilization ability and embryo development of sperm frozen at $-20^{\circ} \mathrm{C}^{\text {a) }}$

\begin{tabular}{|c|c|c|c|c|c|}
\hline & Storage period & $\begin{array}{c}\text { No. of surviving } \\
\text { oocytes (exp) }\end{array}$ & $\begin{array}{l}\text { No. of fertilized } \\
\text { oocytes }(\%)^{*}\end{array}$ & $\begin{array}{c}\text { No. of 2-cell } \\
\text { embryos }(\%)^{\triangle}\end{array}$ & $\begin{array}{c}\text { No. of } \\
\text { blastocysts }(\%)^{\triangle}\end{array}$ \\
\hline Fresh & & $128(3)$ & $122(95.3 \%)$ & $109(89.3 \%)$ & $76(62.3 \%)$ \\
\hline \multirow[t]{2}{*}{ Freezing } & 1 month & $142(4)$ & $121(85.2 \%)^{\#}$ & $105(86.7 \%)$ & $56(46.3 \%)^{\#}$ \\
\hline & 6 months & $138(4)$ & $67(48.5 \%)^{\#}$ & $42(62.7 \%)^{\#}$ & $19(28.4 \%)^{\#}$ \\
\hline
\end{tabular}

a) *, The percentage is based on the number of surviving oocytes; $\triangle$, the percentage is based on the number of fertilized oocytes; \#, significantly different when compared to the fresh group, $P<0.05$. 
present study, we analyzed the karyotypes of the zygotes derived from spermatozoa preserved at $-20^{\circ} \mathrm{C}$. In the control group, two intact haploid groups of metaphase chromosomes were observed. Multiple chromosome fragments were observed in a male pronucleus in a one-cell zygote derived from frozen sperm (Figure 1B; sample was from group of frozen at $-20^{\circ} \mathrm{C}$ for 1 month).

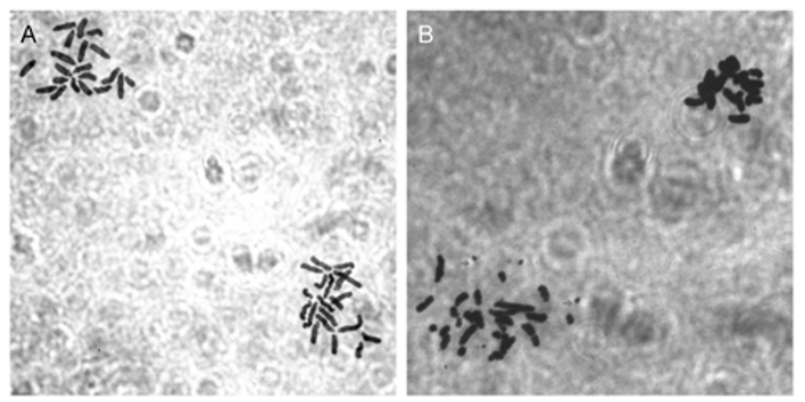

Figure 1 The chromosomes of one-cell zygotes derived from fresh and freezing sperm mitotically arrested by colcemid treatment. A, Normal zygote chromosome karyotype showing two groups of 20 integral chromosomes, one from the oocyte and one from the sperm. B, Chromosomes from a zygote fertilized by a sperm frozen at $-20^{\circ} \mathrm{C}$ for 1 month. Several chromosome fragments (arrows) can be seen. (Number of samples: fresh, $n=21$; frozen at $-20^{\circ} \mathrm{C}$ for 1 month, $n=28$; for 6 months, $n=25$ ).

Of the 21 oocytes injected with fresh spermatozoa that were examined, $20(95.2 \%)$ had normal chromosomes in both pronuclei. Of the 28 zygotes produced from frozen sperm (after a 1-month storage period), 25 (89.3\%) had normal karyotypes and three $(10.7 \%)$ had gross breakages in some of the chromosomes $(P>0.05)$. From 25 zygotes from frozen sperm (after a 6-month storage period), 23 (92\%) had normal karyotype and two (8\%) had gross breakages in some of the chromosomes $(P>0.05)$.

\subsection{Comparison of DNA methylation patterns in pre- implantation embryos}

When DNA methylation patterns in preimplantation embryos were compared, we found that fertilization capacity and embryo development was maintained after short-term storage at $-20^{\circ} \mathrm{C}$ while they were both compromised after long-term storage. Therefore, we choose the short-term storage sperm (1 month) to investigate embryo epigenetic reprogramming.

DNA methylation is an enzyme-mediated process that includes the transfer of a methyl group from S-adenosylmethionine to the $\mathrm{C} 5$ positions of the cytosine in $\mathrm{CpG}$ dinucleotides by different categories of methyltransferases. DNA methylation is subject to dynamic changes during the embryo preimplantation stages. First, in the pronuclear stage, the paternal genome undergoes active DNA demethylation, then the embryo maintains low DNA methylation status until the blastocyst stage when de novo methylation begins [32-35]. In the present study, we used a well-documented 5-me- thylcytidine antibody to visualize the methylation patterns during the different stages $[32,36]$. We found that the dynamic DNA methylation patterns in embryos from the fresh and frozen sperm groups were similar (Figure 2).

\subsection{Distribution patterns of $\mathbf{H 3 K 4 - T r i M ~ i n ~ p r e i m - ~}$ plantation embryos}

Sperm chromatin is decondensed immediately after fertilization, then protamines are replaced by histones [37]. Thereafter, the male pronucleus undergoes dynamic histone H3 methylation [38]. At the pronuclear stage, only the female pronucleus showed H3K4-TriM staining while the male pronucleus showed a complete lack of staining (Figure 3 A1, A2, a1 and a2). The level of H3K4-TriM changed dynamically between the two-cell and blastocyst stages; however, no statistical difference in the temporal distribution pattern of H3K4-TriM between embryos from the control and frozen groups was observed.

\subsection{Dynamic AC-H4K12 modification in preimplan- tation embryos}

At the pronuclear stage, the pattern of AcH4K12 staining was uniform in male and female pronuclei in zygotes from the fresh and frozen sperm groups (Figure 4A1, A2, a1, and a2). As the preimplantation embryos developed, they displayed a homogeneous pattern of AcH4K12 staining in the interphase in both the control and frozen sperm groups (Figure 4B1, B2-E1, E2, b1, b2-e1, and e2). At the blastocyst stage, AcH4K12 staining was less intense in the inner cell mass (ICM) compared to in the trophectoderm cell (TE) (Figure 4F1, F2, f1, and f2). Thus, as with H3K4-TriM, there was no difference in the AcH4K12 distribution patterns from the zygote to blastocyst stages in the two groups.

\section{Discussion}

In the present study, we reported that sperm preserved at $-20^{\circ} \mathrm{C}$ for a short time (1 month) maintained substantial fertilization ability and could support embryo development to the blastocyst stage. Our results confirm the findings of Wakayama et al. [11] who explored simple methods using various physiological solutions (CZB, PBS or isotonic saline) to preserve sperm at $-20^{\circ} \mathrm{C}$. We further demonstrated that this method did not destroy chromosome integrity in the spermatozoa. For the first time, we investigated epigenetic reprogramming, including DNA methylation, histone $\mathrm{H} 3 \mathrm{~K} 4$ trimethylation and histone H4K12 acetylation, in preimplantation embryo derived from such spermatozoa. The epigenetic reprogramming dynamically changed from the pronuclear to blastocyst stages in the control group (using fresh sperm) and in the frozen sperm group; however, no significant difference in temporal distribution was ob- 

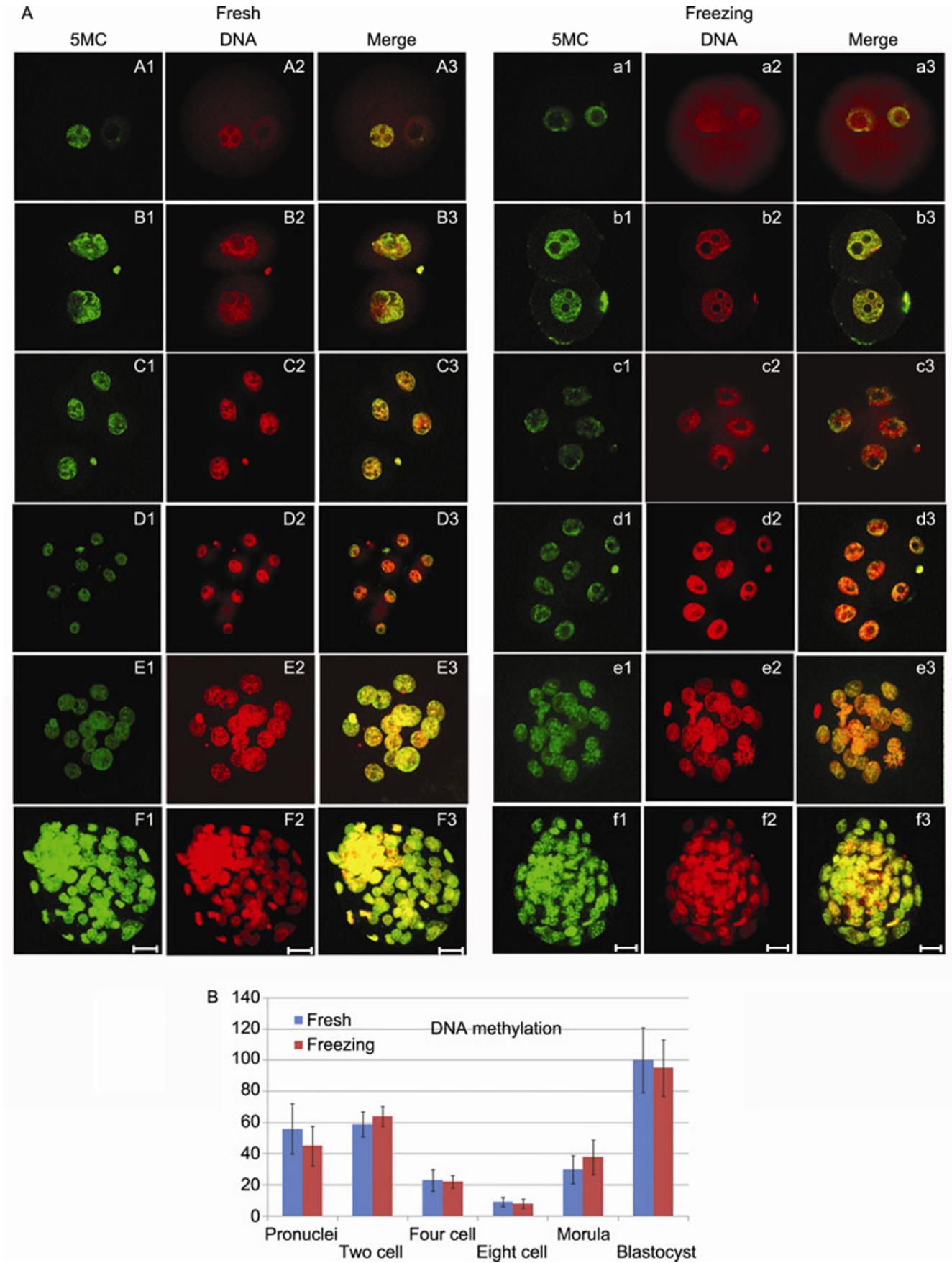

Figure 2 DNA methylation patterns in preimplantation embryos. A, Patterns of DNA methylation (green, A1-F1 and a1-f1) and DNA (red, A2-F2 and a2-f2) in fresh and frozen sperm groups. DNA demethylation pattern in zygotes (A1 and a1), in the two-cell (B1 and b1), four-cell (C1 and c1), and eight-cell (D1 and d1) embryos, and in morula (E1 and e1) and blastocysts (F1 and f1). B, Total nuclear DNA methylation intensities in embryos from the fresh and frozen sperm groups quantified using the ImageJ software. Each column represents the mean value of the intensities averaged on a per embryo basis $(P>0.05)$. At least 20 samples were processed for each embryo stage and the experiments were replicated independently at least three times. Scale bars, $20 \mu \mathrm{m}$.

served between the two groups.

The protocol that is used most to preserve spermatozoa is to freeze the sperm in $\mathrm{LN}_{2}$ with the protection of cryoprotectants. Cryoprotectants provide an energy source for spermatozoa and maintain the osmotic pressure by forming hydrogen bonds with membrane phospholipids and sugars.
This reduces membrane damage and minimizes membrane destabilization during freezing and thawing [39]. This method can preserve spermatozoa for a long time and, after thawing, sperm motility and fertilization ability is restored. However, the method requires the continual supplementation of $\mathrm{LN}_{2}$ and presents safety problems for transportation. 

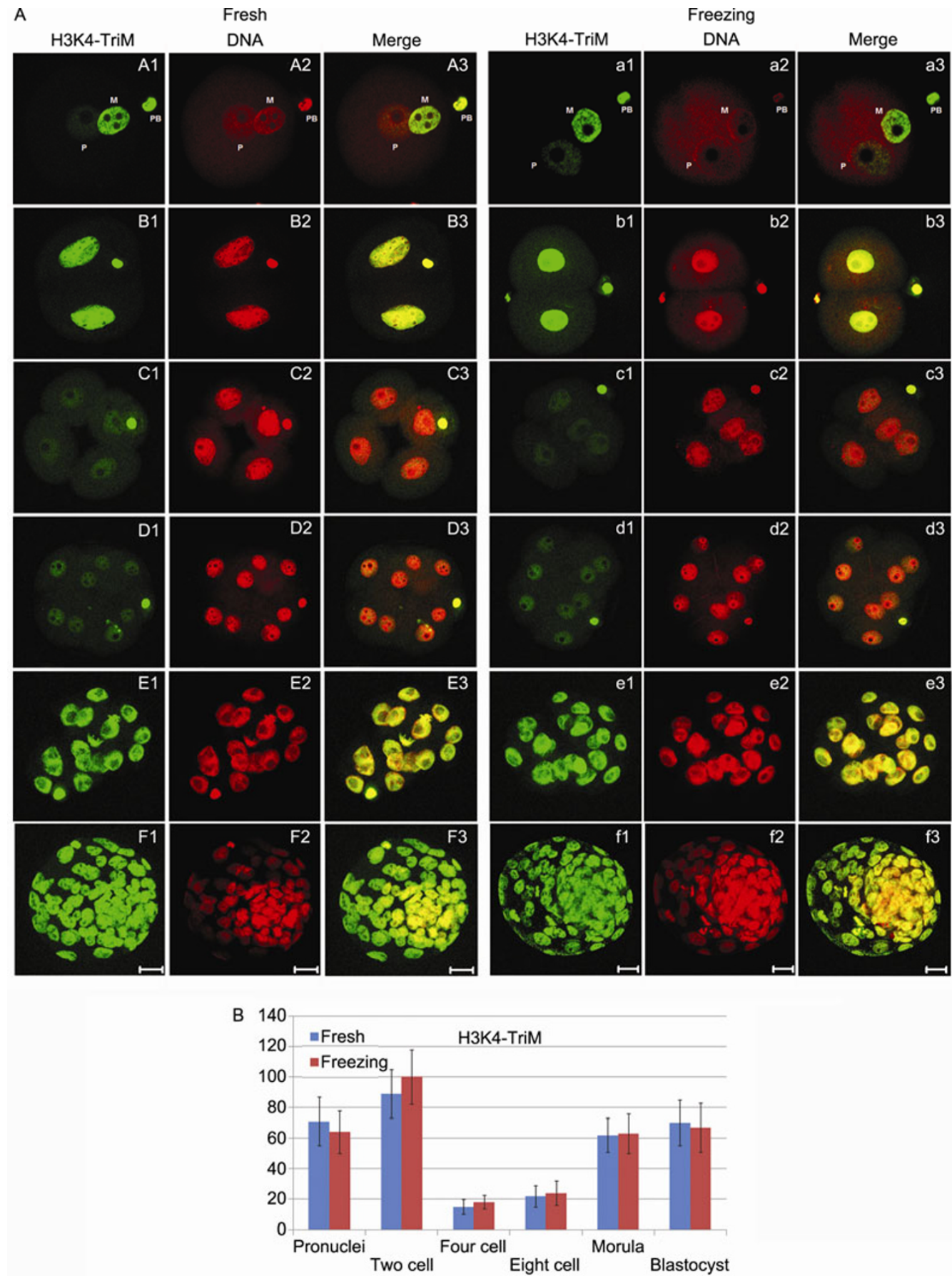

Figure 3 Distribution patterns of H3K4-TriM in preimplantation embryos. A, Methylation patterns of histone H3 Lysine 4 (H3K4-TriM) in fresh sperm (A1-F1) and frozen sperm derived mouse embryos (a1-f1); H3K4-TriM, green and DNA, red. The staining patterns in the one-cell stage in fresh (A1, A2) and frozen sperm group (a1, a2) embryos at pronuclear stage, and in two-cell (B1, B2 and b1, b2), four-cell (C1, C2 and c1, c2), and eight-cell (D1, D2 and d1, d2) embryos, and in morulae (E1, E2 and e1, e2), and blastocysts (F1, F2 and f1, f2). B, Total nuclear H3K4-TriM intensities in embryos of fresh and frozen sperm groups as quantified using ImageJ software. Each column represents the mean value of the intensities averaged on a per embryo basis. At least 20 samples were processed for each embryo stage and the experiments were replicated independently at least three times. Scale bars, $20 \mu \mathrm{m}$.

The motility of thawed sperm seems to be less essential after the application of ICSI. ICSI requires only a single sperm to fulfill fertilization and it is not necessary for the spermatozoa to be motile or alive as long as the nuclei are 'intact' in terms of reproductive and genetic potential [40].
The unique chromatin structure of sperm (highly condensed chromatin and protamines that are extensively cross-linked by disulfide bonds) makes it resistant to freezing and thawing injury [41]. Accordingly, many alternative sperm preservation methods have been explored. 


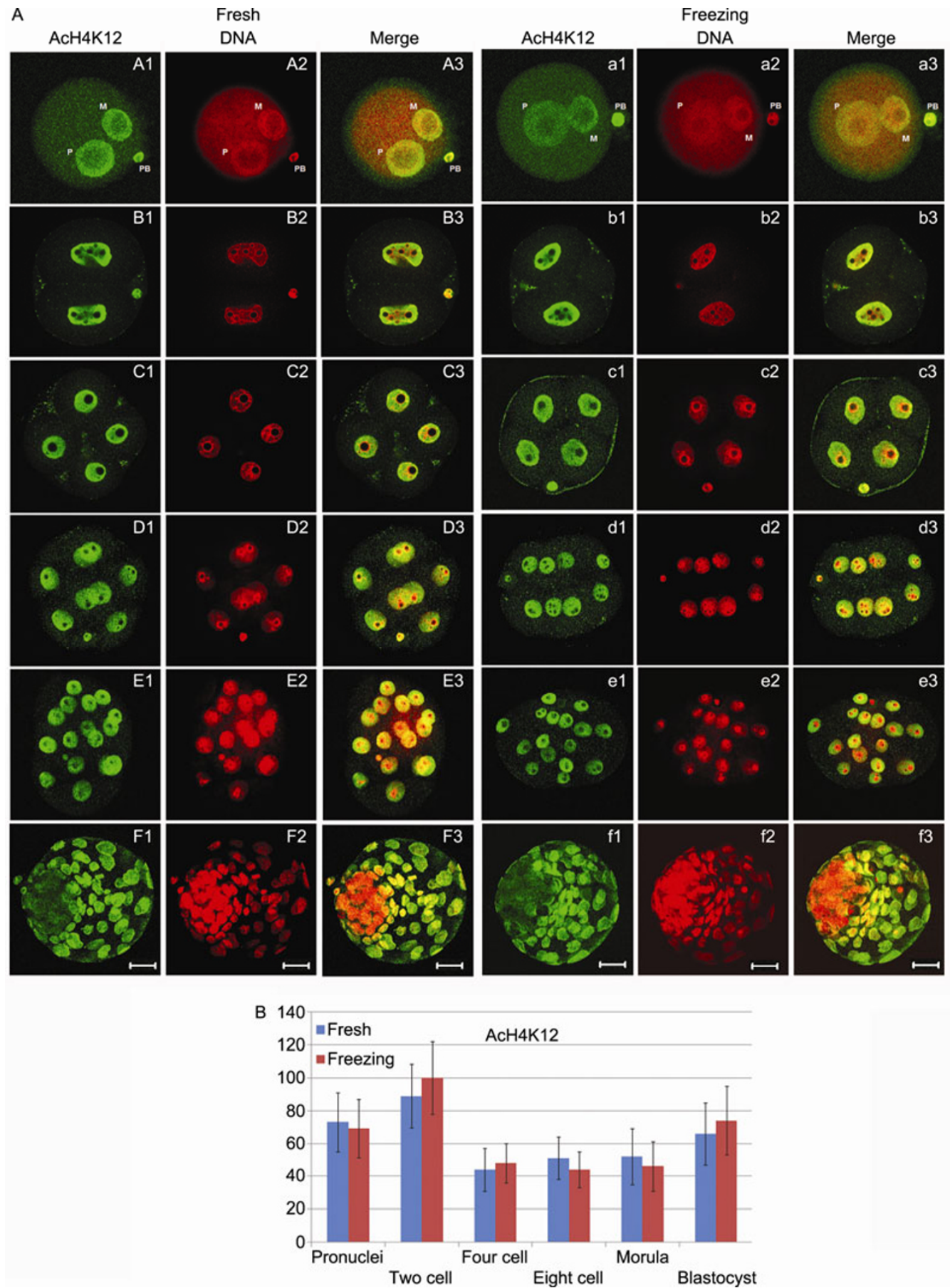

Figure 4 Dynamic AcH4K12 modification in preimplantation embryos. A, Acetylation patterns of histone H4K12 in embryos derived from fresh (A1-F1) and frozen sperm (a1-f1); AcH4K12, green and DNA, red. The staining patterns in one-cell in fresh (A1, A2) and frozen sperm group (a1, a2) embryos at pronuclear stage, and in two-cell (B1, B2 and b1, b2), four-cell (C1, C2 and c1, c2), and eight-cell (D1, D2 and d1, d2) embryos, and in morulae (E1, E2 and e1, e2), and blastocysts (F1, F2 and f1, f2). B, Total nuclear acetylation of histone H4K12 intensities in embryos from the fresh and frozen sperm groups as quantified using ImageJ software. Each bar represents the mean value of the intensities averaged on a per embryo basis. At least twenty samples were processed for each embryo stage and the experiments were replicated independently at least three times. Scale bars, $20 \mu \mathrm{m}$.

Some studies of these methods have focused mainly on the thawed sperm's efficiency for fertilization and embryo development; others have focused on the integrity of the DNA and chromosomes [42]. None of the studies investigated the epigenetics that would be affected by environment [43].
The developmental program of embryogenesis is controlled by both genetic and epigenetic mechanisms. Epigenetic marks are enzyme-mediated chemical modifications of DNA and of its associated chromatin proteins. Although they do not alter the primary sequence of DNA, they play 
important roles in regulating genome function and development. Genome-wide epigenetic reprogramming occurs in the early embryo beginning in the zygote immediately after fertilization and extending to the blastocyst stage of preimplantation development.

DNA methylation is an important epigenetic event and plays a vital role in regulating chromatin structure and gene expression in many developmental processes including gene imprinting, $\mathrm{X}$ chromosome inactivation and embryogenesis [44]. Defective DNA methylation in the early embryo can give rise to aberrant growth and developmental disorders [45]. After fertilization a rapid paternal-specific active demethylation is observed. Thereafter, there is a step-wise decline in methylation until the morula stage and the de novo methylation occurs at blastocyst stage [35,36]. In our experiments, the dynamic process of active demethylation of the paternal genome and de novo methylation at the blastocyst stage was detected in the control group and in the frozen sperm group (Figure 2A1-F1 and a1-f1).

The modification of core histones is another important epigenetic modification to the chromatin which regulates transcription and silencing of a wide range of genes [46,47]. Histone acetylation and deacetylation have been shown to determine the transcriptional activity of the chromatin [48]. DNA methylation and histone modification do not act alone, but interact with one another. For instance, at the blastocyst stage, DNA methylation staining in the ICM was more intense than that in the TE (Figure 2F1, F2, f1, and f2). Coincidentally, AcH4K12 is at a lower level in the ICM than in the TE at this stage (Figure 4F1, F2, f1, and f2). Methylation of the Lys4 residue of $\mathrm{H} 3$ (H3K4) has been associated with active gene expression, whereas methylation of the Lys9 of H3 (H3-K9) has been associated with transcriptional silencing [49]. The histone modifications that are the epigenetic marks that control the developmental pattern of the preimplantation mouse embryo are impacted by many factors, such as the status of the sperm, oocyte quality, and environmental influences (in vitro culture, manipulation) [50]. In the present study, the patterns of AcH4K12 and H3K4TriM in the preimplantation embryos from the fresh and frozen sperm groups showed no significant differences between the two groups. These findings suggested that the simple sperm preservation procedure of freezing at $-20^{\circ} \mathrm{C}$ without cryoprotectants was relatively safe (Figures 3 and 4).

In the current study, we used the simple sperm preservation method first reported by Yanagimachi [8] with minor alterations. All that we did was to replace the preserving medium with HEPES-buffered HTF containing 10\% HSA which has been widely used in animal and human in vitro fertilization. HSA is an important supplement not only for in vitro fertilization and embryo culture but also in spermatozoa cryopreservation. It can protect spermatozoa against chromosomal damage during freezing and thawing [19]. Preserving sperm in an ordinary freezer is probably one of the simplest methods and it can be used in nearly all laboratories. Although the fertilization and embryo development ability were impaired compared to the fresh sperm group, the frozen sperm retained acceptable capacity on short-term storage (Table 1). Considering that cryopreservation protocols using $\mathrm{LN}_{2}$ and elaborate technique of ICSI cannot be widely established in a number of laboratories, especially those in developing countries, the method that has been described and tested in this study should be quite a promising one for use in those laboratories.

In summary, we have shown that the simple spermatozoa preservation method described here does not destroy sperm chromosome integrity and does not change the distribution patterns of the DNA methylation and histone modifications for mouse embryos from the zygote to blastocyst stages. These findings suggest that this simple procedure does not alter the epigenetic reprogramming patterns during the development of the preimplantation embryo.

We thank Hou Yi, Ouyang YingChun and Li ShiWen for their technical assistance and Drs. Guo Lei, Qi ShuTao, Zeng Fang for their insightful discussions and help in writing the manuscript. This work was supported by the National Basic Research Program of China (Grant No. 2011CB944501).

1 Polge C. Smith A U, Parkes A S. Revival of spermatozoa after vitrification and dehydration at low temperatures. Nature, 1949, 164 : 666

2 Johnson L A, Aalbers J G, Willems C M, et al. Use of spermatozoa for artificial insemination. I. Fertilizing capacity of fresh and frozen spermatozoa in sows on 36 farms. J Anim Sci, 1981, 52: 1130-1136

3 Gabriel Sanchez-Partida L, Maginnis G, Dominko T, et al. Live rhesus offspring by artificial insemination using fresh sperm and cryopreserved sperm. Biol Reprod, 2000, 63: 1092-1097

4 Kelleher S, Wishart S M, Liu P Y, et al. Long-term outcomes of elective human sperm cryostorage. Hum Reprod, 2001, 16: 26322639

5 Garcia M A, Graham E F. Development of a buffer system for dialysis of bovine spermatozoa before freezing. II. Effect of sugars and sugar alcohols on posthaw motility. Theriogenology, 1989, 31: 1029-1037

6 Tada N, Sato M, Yamanoi J, et al. Cryopreservation of mouse spermatozoa in the presence of raffinose and glycerol. J Reprod Fertil, 1990, 89: 511-516

7 Kimura Y, Yanagimachi R. Mouse oocytes injected with testicular spermatozoa or round spermatids can develop into normal offspring. Development, 1995, 121: 2397-2405

8 Yanagimachi R. Intracytoplasmic injection of spermatozoa and spermatogenic cells: its biology and applications in humans and animals. Reprod Biomed Online, 2005, 10: 247-288

9 Yazawa H, Yanagida K, Hayashi S, et al. The oocyte activation and $\mathrm{Ca}^{2+}$ oscillation-inducing abilities of mouse and human dead (sonicated) spermatozoa. Zygote, 2009, 17: 175-184

10 Yan W, Morozumi K, Zhang J, et al. Birth of mice after intracytoplasmic injection of single purified sperm nuclei and detection of messenger RNAs and microRNAs in the sperm nuclei. Biol Reprod, 2008, 78: 896-902

11 Wakayama T, Whittingham D G, Yanagimachi R. Production of normal offspring from mouse oocytes injected with spermatozoa cryopreserved with or without cryoprotection. J Reprod Fertil, 1998, 112: 11-17

12 Wakayama T, Yanagimachi R. Development of normal mice from 
oocytes injected with freeze-dried spermatozoa. Nat Biotechnol, 1998, 16: 639-641

13 Kusakabe H, Yanagimachi R, Kamiguchi Y. Mouse and human spermatozoa can be freeze-dried without damaging their chromosomes. Hum Reprod, 2008, 23: 233-239

14 Keskintepe L, Pacholczyk G, Machnicka A, et al. Bovine blastocyst development from oocytes injected with freeze-dried spermatozoa. Biol Reprod, 2002, 67: 409-415

15 Kwon I K, Park K E, Niwa K. Activation, pronuclear formation, and development in vitro of pig oocytes following intracytoplasmic injection of freeze-dried spermatozoa. Biol Reprod, 2004, 71: 14301436

16 Sanchez-Partida L G, Simerly C R, Ramalho-Santos J. Freeze-dried primate sperm retains early reproductive potential after intracytoplasmic sperm injection. Fertil Steril, 2008, 89: 742-745

17 Li M W, Biggers J D, Elmoazzen H Y, et al. Long-term storage of mouse spermatozoa after evaporative drying. Reproduction, 2007, 133: 919-929

18 McGinnis L K, Zhu L, Lawitts J A, et al. Mouse sperm desiccated and stored in trehalose medium without freezing. Biol Reprod, 2005, 73: 627-633

19 Li C, Mizutani E, Ono T, et al. Intracytoplasmic sperm injection with mouse spermatozoa preserved without freezing for six months can lead to full-term development. Biol Reprod, 2011, 85: 1183-1190

20 Ogonuki N, Mochida K, Miki H, et al. Spermatozoa and spermatids retrieved from frozen reproductive organs or frozen whole bodies of male mice can produce normal offspring. Proc Natl Acad Sci USA, 2006, 103: 13098-13103

21 Fleming T P, Kwong W Y, Porter R, et al. The embryo and its future. Biol Reprod, 2004, 71: 1046-1054

22 Feng S, Jacobsen S E, Reik W. Epigenetic reprogramming in plant and animal development. Science, 2010, 330: 622-627

23 Ballestar, E. An introduction to epigenetics. Adv Exp Med Biol, 2011, 711: 1-11

24 Kimura Y, Yanagimachi R. Intracytoplasmic sperm injection in the mouse. Biol Reprod, 1995, 52: 709-720

25 Yoshida N, Perry A C F. Piezo-actuated mouse intracytoplasmic sperm injection (ICSI). Nat Protoc, 2007, 2: 296-304

26 Kamiguchi Y, Mikamo K. An improved, efficient method for analyzing human sperm chromosomes using zona-free hamster ova. Am J Hum Genet, 1986, 38: 724-740

27 Akiyama T, Nagata M, Aoki F. Inadequate histone deacetylation during oocyte meiosis causes aneuploidy and embryo death in mice. Proc Natl Acad Sci USA, 2006, 103: 7339-7344

28 Kusakabe H, Szczygiel M A, Whittingham D G, et al. Maintenance of genetic integrity in frozen and freeze-dried mouse spermatozoa. Proc Natl Acad Sci USA, 2001, 98: 13501-13506

29 Kaneko T, Whittingham D G, Yanagimachi R. Effect of $\mathrm{pH}$ value of freeze-drying solution on the chromosome integrity and developmental ability of mouse spermatozoa. Biol Reprod, 2003, 68: 136-139

30 Kim J M, Ogura A, Nagata M, et al. Analysis of the mechanism for chromatin remodeling in embryos reconstructed by somatic nuclear transfer. Biol Reprod, 2002, 67: 760-766

31 Lacham-Kaplan O, Shaw J, Sanchez-Partida L G, et al. Oocyte activation after intracytoplasmic injection with sperm frozen without cryoprotectants results in live offspring from inbred and hybrid mouse strains. Biol Reprod, 2003, 69: 1683-1689

32 Santos F, Hendrich B, Reik W, et al. Dynamic reprogramming of DNA methylation in the early mouse embryo. Dev Biol, 2002, 241: 172-182

33 Mayer W, Niveleau A, Walter J, et al. Demethylation of the zygotic paternal genome. Nature, 2000, 403: 501-502

34 Dean W, Santos F, Stojkovic M, et al. Conservation of methylation reprogramming in mammalian development: aberrant reprogramming in cloned embryos. Proc Natl Acad Sci USA, 2001, 98: 13734-13738

35 Metivier R, Gallais R, Tiffoche C, et al. Cyclical DNA methylation of a transcriptionally active promoter. Nature, 2008, 452: 45-50

36 Zaitseva I, Zaitsev S, Alenina N, et al. Dynamics of DNAdemethylation in early mouse and rat embryos developed in vivo and in vitro. Mol Reprod Dev, 2007, 74: 1255-1261

37 Lepikhov K, Walter J. Differential dynamics of histone H3 methylation at positions $\mathrm{K} 4$ and $\mathrm{K} 9$ in the mouse zygote. BMC Dev Biol, 2004, 4: 12

38 Lachner M, Jenuwein T. The many faces of histone lysine methylation. Curr Opin Cell Biol, 2002, 14: 286-298

39 Strauss G, Schurtenberger P, Hauser H. The interaction of saccharides with lipid bilayer vesicles: stabilization during freezethawing and freeze-drying. Biochim Biophys Acta, 1986, 858: 169180

40 Yan W, Morozumi K, Zhang J, et al. Birth of mice after intracytoplasmic injection of single purified sperm nuclei and detection of messenger RNAs and MicroRNAs in the sperm nuclei. Biol Reprod, 2008, 78: 896-902

41 Miller D, Brinkworth M, Iles D. Paternal DNA packaging in spermatozoa: more than the sum of its parts? DNA, histones, protamines and epigenetics. Reproduction, 2010, 139: 287-301

42 Yildiz C, Ottaviani P, Law N, et al. Effects of cryopreservation on sperm quality, nuclear DNA integrity, in vitro fertilization, and in vitro embryo development in the mouse. Reproduction, 2007, 133: 585-595

43 Feng S, Jacobsen S E, Reik W. Epigenetic reprogramming in plant and animal development. Science, 2010, 330: 622-627

44 Vassena R, Dee Schramm R, Latham K E. Species-dependent expression patterns of DNA methyltransferase genes in mammalian oocytes and preimplantation embryos. Mol Reprod Dev, 2005, 72: 430-436

45 Feil R. Epigenetic asymmetry in the zygote and mammalian development. Int J Dev Biol, 2009, 53: 191-201

46 Klose R J, Zhang Y. Regulation of histone methylation by demethylimination and demethylation. Nat Rev Mol Cell Biol, 2007, 8: 307-318

47 Kouzarides T. Chromatin modifications and their function. Cell, 2007, 128: 693-705

48 Erhardt S, Su I H, Schneider R, et al. Consequences of the depletion of zygotic and embryonic enhancer of zeste 2 during preimplantation mouse development. Development, 2003, 130: 4235-4248

49 Linggi B E, Brandt S J, Sun Z W, et al. Translating the histone code into leukemia. J Cell Biochem, 2005, 96: 938-950

50 Kishigami S, Van Thuan N, Hikichi T, et al. Epigenetic abnormalities of the mouse paternal zygotic genome associated with microinsemination of round spermatids. Dev Biol, 2006, 289: 195-205

Open Access This article is distributed under the terms of the Creative Commons Attribution License which permits any use, distribution, and reproduction in any medium, provided the original author(s) and source are credited. 